\title{
Dixmier traces generated by exponentiation invariant generalised limits
}

\author{
Fedor Sukochev, Alexandr Usachev, and Dmitriy Zanin
}

\begin{abstract}
We define a new class of singular positive traces on the ideal $\mathcal{M}_{1, \infty}$ of $B(H)$ generated by exponentiation invariant generalized limits. We prove that this new class is strictly contained in the class of all Dixmier traces. We also prove a Lidskii-type formula for this class of traces.
\end{abstract}

Mathematics Subject Classification (2010). 58B34, 46L52.

Keywords. Dixmier traces, measurable elements, exponentiation invariant generalized limits, Lidskii formula.

\section{Introduction and preliminaries}

In the framework of noncommutative geometry the Dixmier traces, originally introduced by J. Dixmier in [7], have become an indispensable tool. These traces are defined via dilation invariant generalized limits $\omega$ on $L_{\infty}(0, \infty)$. Depending on a specific problem, various additional conditions on $\omega$ are imposed [1]-[7], [13], [16]-[19].

For instance, important formulae of noncommutative geometry, involving heat kernel asymptotics and generalised $\zeta$-functions (see e.g. [3], [2]) were established for Dixmier traces $\operatorname{Tr}_{\omega}$, provided that $\omega$ was additionally chosen to be exponentiation invariant. Indeed, in [3] these formulae were proven for Dixmier traces, generated by Cesàro and exponentiation invariant generalized limits $\omega$. In [2] these assumptions were relaxed to dilation and exponentiation invariance (see the definition below).

We thank the referee for very careful reading of the manuscript and suggesting a number of improvements. In particular, the statement of Theorem 16 is due to the referee.

1.1. Generalized limits. Let $L_{\infty}=L_{\infty}(0, \infty)$ be the space of all real-valued bounded Lebesgue measurable functions on $(0, \infty)$ equipped with the norm

$$
\|x\|_{L_{\infty}}:=\operatorname{esssup}_{t>0}|x(t)|
$$


A normalized positive linear functional on $L_{\infty}$ which equals the ordinary limit on convergent (at infinity) sequences is called a generalized limit.

Define the following linear transformations on $L_{\infty}$ :

1. Translations

$$
\left(T_{l} x\right)(t):=x(t+l), \quad l>0, x \in L_{\infty} .
$$

2. Dilations

$$
\left(\sigma_{\frac{1}{\beta}} x\right)(t):=x(\beta t), \quad \beta>0, x \in L_{\infty}
$$

3. Exponentiations

$$
\left(P^{a} x\right)(t)=x\left(t^{a}\right), \quad a>0, x \in L_{\infty}
$$

A generalized limit $\omega$ on $L_{\infty}$ is said to be dilation invariant if

$$
\omega\left(\sigma_{s} x\right)=\omega(x) \text { for every } x \in L_{\infty} \text { and every } s>0 \text {. }
$$

Similarly we define translation and exponentiation invariant generalized limits.

Translation and dilation invariant generalized limits on $L_{\infty}$ were studied in [14], [16], [18] in terms of Banach-type sublinear functionals.

It was proven in [18], Theorem 13 (see also [14], [16]) that for every translation invariant generalized limit $\omega$ on $L_{\infty}$ and any uniformly continuous function $x \in L_{\infty}$ we have

$$
\omega(x) \leq p_{T}(x):=\lim _{t \rightarrow+\infty} \sup _{h \geq 0} \frac{1}{t} \int_{0}^{t} x(s+h) d s .
$$

It should be pointed out that the condition that $x$ is uniformly continuous is essential (see e.g. [14], Remark 5.6(1)).

Remark 1. For every translation invariant generalized limit $\gamma$ on $L_{\infty}$ the composition $\gamma \circ \exp$ defines dilation invariant generalized limit (see [18], Remark 16, for details). Similarly, $\gamma \circ \exp \circ \exp$ defines an exponentiation invariant generalized limit.

Conversely, if $\omega$ is an exponentiation invariant generalized limit, then $\omega \circ \log$ and $\omega \circ \log \circ \log$ are dilation and translation invariant generalized limits, respectively.

Using Remark 1 the result similar to (1) was proven for dilation invariant generalized limit and any function $x \in L_{\infty}$ such that $x \circ \exp$ is uniformly continuous [18], Theorem 17.

1.2. Dixmier traces. Let $B(H)$ be an algebra of all bounded linear operators on a separable Hilbert space $H$ equipped with the uniform norm and let Tr be the standard trace.

For every operator $T \in B(H)$ a generalized singular value function $\mu(T)$ is defined by the formula

$$
\mu(t, T)=\inf \{\|T p\| \mid p \text { is a projection in } B(H) \text { with } \operatorname{Tr}(1-p) \leq t\} .
$$


For a compact operator $T$, it can be proven that $\mu(k-1, T)$ is the $k$-th largest eigenvalue of an operator $|T|=\left(T^{*} T\right)^{1 / 2}, k \in \mathbb{N}$.

Recall that for any $T \in B(H)$ the distribution function of $T$ is defined (see [8]) by setting

$$
d_{T}(t):=\operatorname{Tr}\left(\chi_{(t, \infty)}(|T|)\right), \quad t>0 .
$$

Here $\chi_{(t, \infty)}(|T|)$ is the spectral projection of $|T|$ corresponding to the interval $(t, \infty)$. By [8], Proposition 2.2, we have

$$
\mu(s, T)=\inf \left\{t \geq 0 \mid d_{T}(t) \leq s\right\},
$$

so we infer that for any operator $T$, the distribution function $d_{T}$ coincides with the (classical) distribution function of $\mu(\cdot, T)$.

Since $B(H)$ is an atomic von Neumann algebra and traces of all atoms are equal to 1 , it follows that $\mu(T)$ is a step function and

$$
\mu(T)=\sum_{n=0}^{\infty} \mu(n, T) \chi_{[n, n+1)} \quad \text { for every } T \in B(H) .
$$

The classical Dixmier ideal (see e.g. [5], [11], [13]) is defined by

$$
\mathcal{M}_{1, \infty}:=\left\{T \mid\|T\|_{\mathcal{M}_{1, \infty}}:=\sup _{t>0} \frac{1}{\log (1+t)} \int_{0}^{t} \mu(s, T) d s<\infty\right\} .
$$

A definition of (Dixmier) traces given in [7] can be restated as follows

$$
\operatorname{Tr}_{\omega}(T):=\omega\left(\frac{1}{\log (1+t)} \int_{0}^{t} \mu(s, T) d s\right), \quad 0 \leq T \in \mathcal{M}_{1, \infty},
$$

where $\omega$ is an arbitrary dilation invariant generalized limit on $L_{\infty}$. We denote the set of all Dixmier traces by $\mathscr{D}$.

1.3. Measurability. The following natural way to generate dilation invariant generalized limits was suggested in [6], Section IV, $2 \beta$. A. Connes observed that for any generalised limit $\gamma$ on $L_{\infty}$ a functional $\omega:=\gamma \circ M$ is a dilation invariant generalized limit on $L_{\infty}$. Here, the operator $M: L_{\infty} \rightarrow L_{\infty}$ is the Cesàro mean of the multiplicative group $\mathbb{R}_{+}$, given by the formula

$$
(M x)(t):=\frac{1}{\log t} \int_{1}^{t} x(s) \frac{d s}{s} .
$$

The subclass $\mathscr{C} \subset \mathscr{D}$ of all Dixmier traces $\operatorname{Tr}_{\omega}$ defined by such $\omega$ 's was termed Connes-Dixmier traces in [13]. It was proven in [19] that $\mathcal{C} \subsetneq D$.

As it was mentioned above, various important formulae of noncommutative geometry were established for dilation and exponentiation invariant generalized limits $\omega$. The former assumption was needed in order that the formula (2) defines a Dixmier trace. 
In the present paper we prove that for every exponentiation invariant generalized limit $\omega$ on $L_{\infty}$ the formula (2) defines a Dixmier trace (see Proposition 5 below). Denote by $\mathscr{D}_{P}$ the set of all Dixmier traces generated by such $\omega$ 's.

The following definitions were motivated by A. Connes [6], IV.2. $\beta$.Definition 7 (see also [13], Definition 3.2).

Definition 2. An operator $T \in \mathcal{M}_{1, \infty}$ is called Dixmier measurable if $\operatorname{Tr}_{\omega}(T)$ takes the same value for all $\operatorname{Tr}_{\omega} \in \mathscr{D}$.

A criterion for an operator $T \in \mathcal{M}_{1, \infty}$ (respectively, a positive operator $T \in$ $\mathcal{M}_{1, \infty}$ ) to be Dixmier measurable can be found in [18], Theorem 21 (respectively, [13]). The crucial point in these proof is played by the fact that the function

$$
t \rightarrow \frac{1}{\log \left(1+e^{t}\right)} \int_{0}^{e^{t}} \mu(s, T) d s
$$

is uniformly continuous.

Definition 3. An operator $T \in \mathcal{M}_{1, \infty}$ is called $\mathscr{D}_{P}$-measurable if $\operatorname{Tr}_{\omega}(T)$ takes the same value for all $\operatorname{Tr}_{\omega} \in \mathscr{D}_{P}$.

Using Remark 1 we can easily write down the Banach-type sublinear functional for exponentiation invariant generalized limits. However, we cannot gain any results about $\mathscr{D}_{P}$-measurability on this way, since there exist $0 \leq T \in \mathcal{M}_{1, \infty}$ such that the function

$$
t \rightarrow \frac{1}{\log \left(1+e^{e^{t}}\right)} \int_{0}^{e^{e^{t}}} \mu(s, T) d s
$$

is not uniformly continuous. As an example of such $T$ we may take the operator $T_{0}$ defined in Theorem 8 below.

We prove (see Theorem 8 below) that the class of $\mathscr{D}_{P}$-measurable operators is strictly wider then the class of Dixmier measurable operators. In particular, $\mathscr{D}_{P}$ is a proper subset of $\mathscr{D}$.

1.4. Lidskii formula for Dixmier traces. The classical Lidskii Theorem asserts that

$$
\operatorname{Tr}(T)=\sum_{n \geq 0} \lambda(n, T)
$$

for any trace class operator $T$. Here $\{\lambda(n, T)\}_{n \geq 0}$ is the sequence of eigenvalues of $T$ (counting with algebraic multiplicities), taken in an arbitrary order. This arbitrariness of the order is due to the absolute convergence of the series $\sum_{n \geq 0}|\lambda(n, T)|$. In particular, we can choose a decreasing order of $|\lambda(n, T)|$.

The core difference of this situation with the setting of Dixmier traces living on the ideal $\mathcal{M}_{1, \infty}$ is that the series $\sum_{n \geq 0}|\lambda(n, T)|$ diverges for $T \in \mathcal{M}_{1, \infty}$ (see [17] for a detailed explanation). 
The following theorem gives an analogue of the Lidskii formula for ConnesDixmier traces.

Theorem 4 ([17], Theorem 2). Let $\operatorname{Tr}_{\omega}$ be a Connes-Dixmier trace on $\mathcal{M}_{1, \infty}$. We have

$$
\operatorname{Tr}_{\omega}(T)=\omega\left(\frac{1}{\log (1+t)} \sum_{\lambda \in \sigma(T):|\lambda|>1 / t} \lambda\right), \quad T \in \mathcal{M}_{1, \infty},
$$

where $\sigma(T)$ denotes the spectrum of an operator $T$.

It was also shown in [17], Theorem 5, that there exists a Dixmier trace $\operatorname{Tr}_{\omega}$ such that the formula (3) does not hold.

One of the main results of the present paper (see Theorem 15) asserts that the formula (3) holds for every Dixmier trace $\operatorname{Tr}_{\omega} \in \mathscr{D}_{P}$.

\section{Dixmier traces generated by exponentiation invariant generalised limits}

The following proposition is an analogue of [11], Proposition 10, for exponentiation invariant generalised limits. It shows that every exponentiation invariant generalised limit generates a Dixmier trace.

Proposition 5. For every exponentiation invariant generalised limit $\omega$ on $L_{\infty}$ the weight

$$
\operatorname{Tr}_{\omega}(T):=\omega\left(\frac{1}{\log (1+t)} \int_{0}^{t} \mu(s, T) d s\right), \quad 0 \leq T \in \mathcal{M}_{1, \infty},
$$

extends to a non-normal trace on $\mathcal{M}_{1, \infty}$.

Proof. By the construction of $\operatorname{Tr}_{\omega}$ we only need to prove its additivity on the positive cone of $\mathcal{M}_{1, \infty}$.

Let $0 \leq A, B \in \mathcal{M}_{1, \infty}$. By [8], Theorem 4.4 (ii), we have

$$
\int_{0}^{t / 2} \mu(s, A)+\mu(s, B) d s \leq \int_{0}^{t} \mu(s, A+B) d s \leq \int_{0}^{t} \mu(s, A)+\mu(s, B) d s
$$

for every $t>0$.

Thus, using the positivity of $\omega$, we obtain

$$
\operatorname{Tr}_{\omega}(A+B) \leq \operatorname{Tr}_{\omega}(A)+\operatorname{Tr}_{\omega}(B)
$$

for every generalised limit $\omega$. 
On the other hand, we have

$$
\begin{aligned}
\operatorname{Tr}_{\omega}(A)+\operatorname{Tr}_{\omega}(B) & =\omega\left(\frac{1}{\log (1+t)} \int_{0}^{t} \mu(s, A)+\mu(s, B) d s\right) \\
& \leq \omega\left(\frac{1}{\log (1+t)} \int_{0}^{2 t} \mu(s, A+B) d s\right) .
\end{aligned}
$$

Since for every $\varepsilon>0$ we have $2 t \leq t^{1+\varepsilon}$ (for $t$ large enough) and

$$
\log \left(1+t^{1+\varepsilon}\right) \leq(1+\varepsilon) \log (1+t),
$$

the following estimate holds:

$$
\begin{aligned}
\operatorname{Tr}_{\omega}(A)+\operatorname{Tr}_{\omega}(B) & \leq \omega\left(\frac{1}{\log (1+t)} \int_{0}^{2 t} \mu(s, A+B) d s\right) \\
& \leq(1+\varepsilon) \omega\left(\frac{1}{\log \left(1+t^{1+\varepsilon}\right)} \int_{0}^{t^{1+\varepsilon}} \mu(s, A+B) d s\right) \\
& \leq(1+\varepsilon) \omega\left(\frac{1}{\log (1+t)} \int_{0}^{t} \mu(s, A+B) d s\right) \\
& =(1+\varepsilon) \operatorname{Tr}_{\omega}(A+B) .
\end{aligned}
$$

Since $\varepsilon>0$ can be chosen arbitrary small, we conclude that $\operatorname{Tr}_{\omega}(A)+\operatorname{Tr}_{\omega}(B) \leq$ $\operatorname{Tr}_{\omega}(A+B)$.

It is obvious from the definition of $\operatorname{Tr}_{\omega}$ in Proposition 5 that every such functional is fully symmetric (see e.g. [5], [11]). By the main result of [11] the set of all fully symmetric functionals on $\mathcal{M}_{1, \infty}$ coincides with the set of all Dixmier traces, that is with the set $\mathscr{D}$. Thus, singular traces generated by an exponentiation invariant generalised limit $\omega$ are Dixmier traces. Next, we show that the class of Dixmier measurable operators is strictly wider then the class of $\mathscr{D}_{P}$-measurable operators.

First, we state two auxiliary lemmas.

Lemma 6 ([14], Example 5.6(1)). Let $x \in L_{\infty}$ be a locally Riemann integrable function. If $x$ is a periodic function and its period is $l>0$, then

$$
\gamma(x)=\frac{1}{l} \int_{0}^{l} x(s) d s,
$$

for every translation invariant generalized limit $\gamma$ on $L_{\infty}$.

The following lemma is an analogue of [19], Lemma 1.2, for exponentiation invariant generalised limits. 
Lemma 7. For every $T \in \mathcal{M}_{1, \infty}$ and for every exponentiation invariant generalised limit $\omega$, we have

$$
\omega\left(\frac{t \mu(t, T)}{\log (1+t)}\right)=0
$$

Proof. Since $\omega$ is an exponentiation invariant generalised limit, it follows that

$$
\omega\left(\frac{1}{\log (1+t)} \int_{0}^{t / 2} \mu(s, T) d s\right)=\omega\left(\frac{1}{\log \left(1+t^{1+\varepsilon}\right)} \int_{0}^{\frac{t^{1+\varepsilon}}{2}} \mu(s, T) d s\right)
$$

for every $\varepsilon>0$. For every fixed $\varepsilon>0$ we have $\frac{t^{1+\varepsilon}}{2} \geq t$ (for $t$ large enough) and

$$
\log \left(1+t^{1+\varepsilon}\right) \leq(1+\varepsilon) \log (1+t) \quad \text { for all } t>0 .
$$

Hence,

$$
\omega\left(\frac{1}{\log (1+t)} \int_{0}^{t / 2} \mu(s, T) d s\right) \geq \frac{1}{1+\varepsilon} \cdot \omega\left(\frac{1}{\log (1+t)} \int_{0}^{t} \mu(s, T) d s\right) .
$$

Since $\varepsilon$ is arbitrary, it follows that

$$
\omega\left(\frac{1}{\log (1+t)} \int_{0}^{t / 2} \mu(s, T) d s\right)=\omega\left(\frac{1}{\log (1+t)} \int_{0}^{t} \mu(s, T) d s\right) .
$$

Consequently, since $\mu(T)$ is decreasing, it follows that

$$
0=\omega\left(\frac{1}{\log (1+t)} \int_{t / 2}^{t} \mu(s, T) d s\right) \geq \omega\left(\frac{t \mu(t, T)}{2 \log (1+t)}\right) \geq 0 .
$$

In view of the main result of [13] the set of all positive $\mathscr{D}$-measurable and the set of all positive $\mathcal{C}$-measurable elements coincide. The following theorem shows that the sets of all positive $\mathscr{D}_{P}$-measurable and all positive $\mathcal{C}$-measurable elements are distinct, in particular, the classes $\mathscr{D}_{P}$ and $\mathscr{C}$ are different.

Theorem 8. There exists a positive Dixmier non-measurable operator $T_{0} \in \mathcal{M}_{1, \infty}$ such that all $\operatorname{Tr}_{\omega} \in D_{P}$ take the same value on $T_{0}$.

Proof. Let $T_{0}$ be a compact operator on $H$ such that

$$
\mu\left(T_{0}\right)=\sup _{k \geq 0} e^{k-e^{k}} \chi_{\left[0,\left\lfloor e^{e^{k}}\right\rfloor\right)} .
$$

Consider a function

$$
z=\sup _{k \geq 0} e^{k-e^{k}} \chi_{\left[0, e^{e^{k}}\right)}
$$

It is easy to see that $\mu\left(T_{0}\right)-z \in L_{1}(0, \infty) \cap L_{\infty}(0, \infty)$. 
For every $e^{e^{n}} \leq t<e^{e^{n+1}}$ we have

$$
\begin{aligned}
\frac{1}{\log (1+t)} \int_{0}^{t} \mu\left(s, T_{0}\right) d s & =\frac{1}{\log (1+t)}\left(\int_{0}^{e^{e^{n}}} z(s) d s+\left(t-e^{e^{n}}\right) \mu\left(t, T_{0}\right)+O(1)\right) \\
& =\frac{1}{\log (1+t)}\left(\sum_{k=1}^{n} \int_{e^{e^{k-1}}}^{e^{e^{k}}} e^{k-e^{k}} d s+t \mu\left(t, T_{0}\right)+O(1)\right) \\
& =\frac{1}{e-1} \frac{e^{n+1}}{\log t}+\frac{t \mu\left(t, T_{0}\right)}{\log (1+t)}+o(1)
\end{aligned}
$$

Now it is easy to check that

$$
\frac{1}{\log (1+t)} \int_{0}^{t} \mu\left(s, T_{0}\right) d s \leq \frac{e^{2}}{e-1}+o(1), \quad t>0
$$

and therefore $T_{0} \in \mathcal{M}_{1, \infty}$. However, the limit

$$
\lim _{t \rightarrow \infty} \frac{1}{\log (1+t)} \int_{0}^{t} \mu\left(s, T_{0}\right) d s
$$

does not exist since by (4),

$$
\lim _{n \rightarrow \infty} \frac{1}{\log \left(1+e^{e^{n}}\right)} \int_{0}^{e^{e^{n}}} \mu\left(s, T_{0}\right) d s=\frac{e}{e-1}
$$

and

$$
\lim _{n \rightarrow \infty} \frac{1}{\log \left(1+\frac{e^{e^{n+1}+e^{e^{n}}}}{2}\right)} \int_{0}^{\frac{e^{e^{n+1}+e^{e^{n}}}}{2}} \mu\left(s, T_{0}\right) d s=\frac{1}{e-1}+\frac{1}{2} .
$$

Thus, $T_{0}$ is not Dixmier measurable (due to Theorem 6.6 of [13]). It remains to show that $T_{0}$ is $\mathscr{D}_{P}$-measurable.

By Lemma 7 we have

$$
\omega\left(\frac{t \mu\left(t, T_{0}\right)}{\log (1+t)}\right)=0
$$

Let

$$
x(t):=\frac{e}{e-1} \sum_{k=0}^{\infty} \frac{e^{k}}{\log t} \chi_{\left[e^{e^{k}}, e^{e^{k+1}}\right)}(t), \quad t>0 .
$$

In view of (4) we only need to prove that all exponentiation invariant generalised limits coincide on $x$. By Remark 1 , it is sufficient to show that all translation invariant generalised limits coincide on $x \circ \exp \circ \exp$.

Since for $n \leq t<n+1$,

$$
(x \circ \exp \circ \exp )(t)=\frac{e}{e-1} \sum_{k=0}^{\infty} \frac{e^{k}}{e^{t}} \chi_{[k, k+1)}(t),
$$


we conclude that the function $x \circ \exp \circ \exp$ is a Riemann integrable periodic function. By Lemma 6, for every translation invariant generalised limit $\gamma$, we have

$$
\gamma(x \circ \exp \circ \exp )=\frac{e}{e-1} \int_{0}^{1} \frac{d s}{e^{s}}=\frac{e}{e-1} \frac{e-1}{e}=1 .
$$

So, by Remark $1, \omega(x)=1$ for every exponentiation invariant generalised limit $\omega$.

Hence,

$$
\operatorname{Tr}_{\omega}\left(T_{0}\right)=\omega\left(\frac{1}{\log (1+t)} \int_{0}^{t} \mu\left(s, T_{0}\right) d s\right)=1
$$

for every exponentiation invariant generalised limit $\omega$.

Corollary 9. The set $\mathfrak{D}_{P}$ is a proper subset of the set of all Dixmier traces.

\section{Lidskii formula for Dixmier traces generated by exponentiation invariant generalised limits}

In the present section we first prove the Lidskii formula for positive operators $T \in$ $\mathcal{M}_{1, \infty}$. Then, using Ringrose's representation [15], Theorems 1, 6, 7, of compact operators, we extend the formula to an arbitrary $T \in \mathcal{M}_{1, \infty}$.

Lemma 10. For every positive $T \in \mathcal{M}_{1, \infty}$ and for every exponentiation invariant generalized limit $\omega$ the following formula holds

$$
\operatorname{Tr}_{\omega}(T)=\omega\left(\frac{1}{\log (1+t)} \int_{0}^{d_{T}(1 / t)} \mu(s, T) d s\right) .
$$

Proof. Let $T$ be a positive operator from $\mathcal{M}_{1, \infty}$ and $\omega$ be an exponentiation invariant generalized limit on $L_{\infty}$. From the proof of [3], Proposition 2.4, we know that

$$
\int_{0}^{t} \mu(s, T) d s \leq \int_{0}^{d_{T}(1 / t)} \mu(s, T) d s+1 .
$$

Dividing both sides by $\log (1+t)$ and applying $\omega$, we obtain

$$
\operatorname{Tr}_{\omega}(T) \leq \omega\left(\frac{1}{\log (1+t)} \int_{0}^{d_{T}(1 / t)} \mu(s, T) d s\right) .
$$

On the other hand, by [3], Lemma 2.3, there exists a constant $C>0$ such that we eventually have

$$
d_{T}\left(\frac{1}{t}\right) \leq C \cdot t \log (1+t) \leq t^{1+\varepsilon}, \quad \varepsilon>0 .
$$


We have

$$
\frac{1}{\log (1+t)}=\frac{1+\varepsilon}{\log (1+t)^{1+\varepsilon}} \leq \frac{1+\varepsilon}{\log \left(1+t^{1+\varepsilon}\right)} .
$$

Hence, by (5) and (6), we obtain

$$
\begin{aligned}
\omega\left(\frac{1}{\log (1+t)} \int_{0}^{d_{T}(1 / t)} \mu(s, T) d s\right) & \leq \omega\left(\frac{1+\varepsilon}{\log \left(1+t^{1+\varepsilon}\right)} \int_{0}^{t^{1+\varepsilon}} \mu(s, T) d s\right) \\
& \leq(1+\varepsilon) \tau_{\omega}(T)
\end{aligned}
$$

since $\omega$ is an exponentiation invariant generalized limit. Since $\varepsilon>0$ is arbitrary, we obtain the converse inequality.

Corollary 11. If $T=T^{*} \in \mathcal{M}_{1, \infty}$, then

$$
\operatorname{Tr}_{\omega}(T)=\omega\left(\frac{1}{\log (1+t)} \sum_{\lambda \in \sigma(T),|\lambda|>1 / t} \lambda\right)
$$

for every exponentiation invariant Dixmier trace $\operatorname{Tr}_{\omega}$.

The following Lemma is an analogue of [18], Lemma 42, for exponentiation invariant generalized limits.

Lemma 12. For every positive $T \in \mathcal{M}_{1, \infty}$ and for every exponentiation invariant generalized limit $\omega$, we have

$$
\omega\left(\frac{1}{t \log (1+t)} d_{T}\left(\frac{1}{t}\right)\right)=0 .
$$

Proof. Fix $0<\varepsilon<1$. By the definition of a distribution function we have

$$
\frac{1}{t}\left(d_{T}\left(\frac{1}{t}\right)-d_{T}\left(\frac{1}{t^{1-\varepsilon}}\right)\right) \leq \sum_{1 / t<\lambda \leq 1 / t^{1-\varepsilon}} \lambda
$$

Dividing both sides by $\log (1+t)$ and applying $\omega$, we obtain

$$
\begin{gathered}
\omega\left(\frac{1}{t \log (1+t)} d_{T}\left(\frac{1}{t}\right)-\omega\left(\frac{1}{t \log (1+t)} d_{T}\left(\frac{1}{t^{1-\varepsilon}}\right)\right)\right. \\
\leq \omega\left(\frac{1}{\log (1+t)} \sum_{1 / t<\lambda \leq 1 / t^{1-\varepsilon}} \lambda\right) .
\end{gathered}
$$

However, using (5) we have

$$
\lim _{t \rightarrow \infty} \frac{1}{t \log (1+t)} d_{T}\left(\frac{1}{t^{1-\varepsilon}}\right) \leq \lim _{t \rightarrow \infty} \frac{1}{t \log (1+t)} t^{(1-\varepsilon)(1+\varepsilon)}=0 .
$$


Since $\omega$ is a generalized limit, it follows that

$$
\omega\left(\frac{1}{t \log (1+t)} d_{T}\left(\frac{1}{t^{1-\varepsilon}}\right)\right)=0 .
$$

Therefore,

$$
\begin{aligned}
\omega\left(\frac{1}{t \log (1+t)} d_{T}\left(\frac{1}{t}\right)\right) & \leq \omega\left(\frac{1}{\log (1+t)} \sum_{1 / t<\lambda \leq 1 / t^{1-\varepsilon}} \lambda\right) \\
& =\omega\left(\frac{1}{\log (1+t)} \sum_{\lambda>1 / t} \lambda\right)-\omega\left(\frac{1}{\log (1+t)} \sum_{\lambda>1 / t^{1-\varepsilon}} \lambda\right) \\
& =\omega\left(\frac{1}{\log (1+t)} \sum_{\lambda>1 / t} \lambda\right)-\omega\left(\frac{1-\varepsilon}{\log \left(1+t^{1-\varepsilon}\right)} \sum_{\lambda>1 / t^{1-\varepsilon}} \lambda\right),
\end{aligned}
$$

where the last equality holds since

$$
\lim _{t \rightarrow \infty} \frac{(1-\varepsilon) \log (1+t)}{\log \left(1+t^{1-\varepsilon}\right)}=1
$$

and since $\omega$ is a generalized limit.

Using exponentiation invariance of $\omega$ and Corollary 11, we obtain

$$
\omega\left(\frac{1}{t \log (1+t)} d_{T}\left(\frac{1}{t}\right)\right) \leq \operatorname{Tr}_{\omega}(T)-(1-\varepsilon) \operatorname{Tr}_{\omega}(T)=\varepsilon \cdot \operatorname{Tr}_{\omega}(T) .
$$

Since $0<\varepsilon<1$ can be chosen arbitrarily small, we conclude that

$$
\omega\left(\frac{1}{t \log (1+t)} d_{T}\left(\frac{1}{t}\right)\right)=0 \text {. }
$$

We need two auxiliary lemmas.

Lemma 13. For every normal operator $T \in \mathcal{M}_{1, \infty}$ and for every exponentiation invariant generalized limit $\omega$, we have

$$
\omega\left(\frac{1}{\log (1+t)} \sum_{\lambda \in \sigma(T):|\lambda|>1 / t} \lambda\right)=\omega\left(\frac{1}{\log (1+t)} \sum_{\substack{\lambda \in \sigma(T):|\operatorname{Re}(\lambda)|>1 / t \\ o r|\operatorname{Im}(\lambda)|>1 / t}} \lambda\right) .
$$

Proof. Consider the difference

$$
\begin{aligned}
\left|\sum_{\lambda \in \sigma(T):|\lambda|>1 / t} \lambda-\sum_{\substack{\lambda \in \sigma(T):|\operatorname{Re}(\lambda)|>1 / t \\
\operatorname{or}|\operatorname{Im}(\lambda)|>1 / t}} \lambda\right| & \leq \sum_{\lambda \in \sigma(T): 1 / t \leq|\lambda| \leq 2 / t}|\lambda| \\
& \leq \frac{2}{t} \sum_{\lambda \in \sigma(T): 1 / t \leq|\lambda| \leq 2 / t} 1 \\
& \leq \frac{2}{t} d_{T}\left(\frac{1}{t}\right) .
\end{aligned}
$$


Hence, by Lemma 12, we have

$$
\begin{aligned}
& \left|\omega\left(\frac{1}{\log (1+t)} \sum_{\substack{\lambda \in \sigma(T):|\lambda|>1 / t\\
}} \lambda\right)-\omega\left(\frac{1}{\log (1+t)} \sum_{\substack{\lambda \in \sigma(T):|\operatorname{Re}(\lambda)|>1 / t \\
\text { or } \operatorname{Im}(\lambda) \mid>1 / t}} \lambda\right)\right| \\
& \leq \omega\left(\frac{2}{t \log (1+t)} d_{T}\left(\frac{1}{t}\right)\right)=0 .
\end{aligned}
$$

Lemma 14. For every normal operator $T \in \mathcal{M}_{1, \infty}$ and for every exponentiation invariant generalized limit $\omega$, we have

$$
\omega\left(\frac{1}{\log (1+t)} \sum_{\substack{\lambda \in \sigma(T):|\operatorname{Re}(\lambda)|>1 / t \\ o r|\operatorname{Im}(\lambda)|>1 / t}} \operatorname{Re}(\lambda)\right)=\omega\left(\frac{1}{\log (1+t)} \sum_{\lambda \in \sigma(T):|\operatorname{Re}(\lambda)|>1 / t} \operatorname{Re}(\lambda)\right)
$$

and

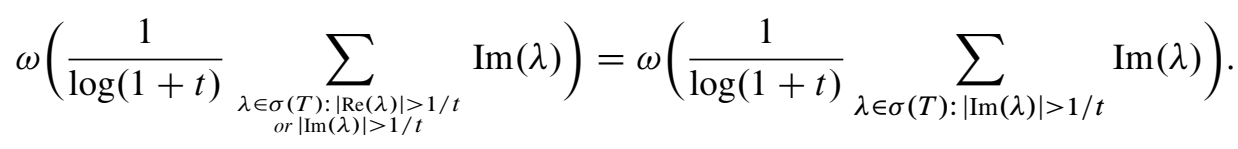

Proof. Consider the difference

$$
\begin{aligned}
\left|\sum_{\lambda \in \sigma(T):|\operatorname{Re}(\lambda)|>1 / t} \operatorname{Re}(\lambda)-\sum_{\substack{\lambda \in \sigma(T):|\operatorname{Re}(\lambda)|>1 / t \\
\text { or }|\operatorname{Im}(\lambda)|>1 / t}} \operatorname{Re}(\lambda)\right| & \leq \sum_{\substack{\lambda \in \sigma(T):|\operatorname{Re}(\lambda)| \leq 1 / t \\
\text { and }|\operatorname{Im}(\lambda)|>1 / t}}|\operatorname{Re}(\lambda)| \\
& \leq \frac{1}{t} \sum_{\substack{\lambda \in \sigma(T):|\operatorname{Im}(\lambda)|>1 / t\\
}} 1 .
\end{aligned}
$$

Since the operator $T$ is normal, it follows that $\operatorname{Im}(\sigma(T))=\sigma(\operatorname{Im}(T))$. Then

$$
\sum_{\lambda \in \sigma(T):|\operatorname{Im}(\lambda)|>1 / t} 1=d_{\operatorname{Im}(T)}\left(\frac{1}{t}\right)
$$

and

$$
\frac{1}{\log (1+t)}\left|\sum_{\lambda \in \sigma(T):|\operatorname{Re}(\lambda)|>1 / t} \operatorname{Re}(\lambda)-\sum_{\substack{\lambda \in \sigma(T):|\operatorname{Re}(\lambda)|>1 / t \\ \operatorname{or}|\operatorname{Im}(\lambda)|>1 / t}} \operatorname{Re}(\lambda)\right| \leq \frac{1}{t \log (1+t)} d_{\operatorname{Im}(T)}\left(\frac{1}{t}\right) .
$$

Applying an exponentiation invariant generalized limit $\omega$ to both sides of the latter expression and using Lemma 12, we obtain the required equality.

The proof of the second equality is similar and is therefore omitted.

The following theorem is an analogue of the Lidskii formula for Dixmier traces generated by exponentiation invariant generalised limits. 
Theorem 15. For every operator $T \in \mathcal{M}_{1, \infty}$ and for every exponentiation invariant generalized limit $\omega$, we have

$$
\operatorname{Tr}_{\omega}(T)=\omega\left(\frac{1}{\log (1+t)} \sum_{\lambda \in \sigma(T):|\lambda|>1 / t} \lambda\right) .
$$

Proof. For every compact operator $T$ there exist a compact normal operator $S$ and a compact quasi-nilpotent operator $Q$ such that $T=S+Q$ and $\sigma(T)=\sigma(S)$ (multiplicities coincide as well) [15], Theorems 1, 6, 7. By the Weil theorem (see e.g. [9], Theorem 3.1), the sequence $|\lambda(T)|$ is majorized by the sequence $\mu(T)$. So, for $T \in \mathcal{M}_{1, \infty}$, we have $S, Q \in \mathcal{M}_{1, \infty}$. By [10], we have $\operatorname{Tr}_{\omega}(Q)=0$ for every quasi-nilpotent operator $Q$ and for every Dixmier trace. Hence, it is sufficient to prove the statement of the theorem for a normal operator $T \in \mathcal{M}_{1, \infty}$.

Let $T \in \mathcal{M}_{1, \infty}$ be normal and let $\omega$ be an exponentiation invariant generalized limit. Since $T$ is normal, it follows that $\operatorname{Re}(\sigma(T))=\sigma(\operatorname{Re}(T))$ and

$$
\sum_{\lambda \in \sigma(T):|\operatorname{Re}(\lambda)|>1 / t} \operatorname{Re}(\lambda)=\sum_{\lambda \in \sigma(\operatorname{Re}(T)):|\lambda|>1 / t} \lambda .
$$

Then by Corollary 11 we obtain

$$
\operatorname{Tr}_{\omega}(\operatorname{Re}(T))=\omega\left(\frac{1}{\log (1+t)} \sum_{\lambda \in \sigma(T):|\operatorname{Re}(\lambda)|>1 / t} \operatorname{Re}(\lambda)\right) .
$$

Similarly, for the operator $\operatorname{Im}(T)$ we obtain

$$
\operatorname{Tr}_{\omega}(\operatorname{Im}(T))=\omega\left(\frac{1}{\log (1+t)} \sum_{\lambda \in \sigma(T):|\operatorname{Im}(\lambda)|>1 / t} \operatorname{Im}(\lambda)\right) .
$$

The assertion of the theorem follows from Lemma 14 and Lemma 13.

We complete this paper with an application to $\zeta$-functions of non-commutative geometry.

Let $\omega$ be a generalized limit on $L_{\infty}$. The functional $\zeta_{\omega}$ defined by the formula

$$
\zeta_{\omega}(T)=\omega\left(\frac{1}{r} \operatorname{Tr}\left(T^{1+1 / r}\right)\right), \quad 0 \leq T \in \mathcal{M}_{1, \infty},
$$

is called a $\zeta$-function residue. It is additive on the positive cone of $\mathcal{M}_{1, \infty}$ and, therefore, it extends to a fully symmetric functional on $\mathcal{M}_{1, \infty}$ [20], Theorem 8.

The following theorem generalizes Theorem 15 of [20] by weakening the assumptions on a generalized limit $\omega$.

Theorem 16. If $\omega$ is an exponentially invariant generalized limit, then

$$
\operatorname{Tr}_{\omega}=\zeta_{\omega \circ \log }
$$


Proof. It is sufficient to prove the equality $\operatorname{Tr}_{\omega}=\zeta_{\omega \circ \log }$ on positive operators $T \in$ $\mathcal{M}_{1, \infty}$.

Define the function $\beta:(0, \infty) \rightarrow(0, \infty)$ by

$$
\beta(t):=\int_{e^{-t}}^{\infty} s d d_{T}(s)
$$

and let

$$
h(r):=\int_{0}^{\infty} e^{-t / r} d \beta(t) .
$$

It follows from spectral theorem that

$$
\operatorname{Tr}\left(T^{1+1 / r}\right)=h(r)
$$

and by the definition of $\zeta$-function we have

$$
\zeta_{\omega \circ \log }(T)=(\omega \circ \log )\left(\frac{1}{r} \operatorname{Tr}\left(T^{1+1 / r}\right)\right)=(\omega \circ \log )\left(\frac{h(r)}{r}\right) .
$$

Since $\omega \circ \log$ is a dilation invariant generalized limit, it follows from the weak*Karamata theorem (see e.g. [3], Theorem 2.2) that

$$
(\omega \circ \log )\left(\frac{h(r)}{r}\right)=(\omega \circ \log )\left(\frac{\beta(t)}{t}\right) .
$$

Hence, we obtain

$$
\begin{aligned}
\zeta_{\omega \circ \log }(T) & =(\omega \circ \log )\left(\frac{\beta(t)}{t}\right) \\
& =(\omega \circ \log )\left(\frac{1}{t} \int_{e^{-t}}^{\infty} s d d_{T}(s)\right) \\
& =\omega\left(\frac{1}{\log t} \int_{1 / t}^{\infty} s d d_{T}(s)\right) \\
& =\operatorname{Tr}_{\omega}(T),
\end{aligned}
$$

where the last equality is due to Theorem 15 .

Acknowledgement. This research was supported by the Australian Research Council.

\section{References}

[1] M.-T. Benameur and T. Fack, Type II non-commutative geometry. I. Dixmier trace in von Neumann algebras. Adv. Math. 199 (2006), 29-87. Zbl 1092.46050 MR 2186918

[2] A. L. Carey, J. Phillips, A. Rennie, and F. A. Sukochev, The Hochschild class of the Chern character for semifinite spectral triples. J. Funct. Anal. 213 (2004), 111-153. Zbl 1058.19002 MR 2069783 
[3] A. Carey, J. Phillips, and F. Sukochev, Spectral flow and Dixmier traces. Adv. Math. 173 (2003), 68-113. Zbl 1015.19003 MR 1954456

[4] A. L. Carey, A. Rennie, A. Sedaev, and F. Sukochev, The Dixmier trace and asymptotics of zeta functions. J. Funct. Anal. 249 (2007), 253-283. Zbl 1128.46022 MR 2345333

[5] A. Carey and F. Sukochev, Dixmier traces and some applications in non-commutative geometry. Uspekhi Mat. Nauk 61 (2006), No. 6, 45-110; English transl. Russian Math. Surveys 61 (2006), 1039-1099. Zbl 1151.46053 MR 2330013

[6] A. Connes, Noncommutative geometry. Academic Press, San Diego, CA, 1994. Zbl 0818.46076 MR 1303779

[7] J. Dixmier, Existence de traces non normales. C. R. Acad. Sci. Paris Sér. A 262 (1966), 1107-1108. Zbl 0141.12902 MR 0196508

[8] T. Fack and H. Kosaki, Generalized $s$-numbers of $\tau$-measurable operators. Pacific $J$. Math. 123 (1986), 269-300. Zbl 0617.46063 MR 840845

[9] I. C. Gohberg and M. G. Krein, Introduction to the theory of linear nonselfadjoint operators. Transl. Math. Monographs 18, American Mathematical Society, Providence, R.I., 1969. Zbl 0181.13504 MR 0246142

[10] N. J. Kalton, Spectral characterization of sums of commutators I. J. Reine Angew. Math. 504 (1998), 115-125. Zbl 0919.47022 MR 1656767

[11] N. J. Kalton, A. A. Sedaev, and F. A. Sukochev, Fully symmetric functionals on a Marcinkiewicz space are Dixmier traces. Adv. Math. 226 (2011), 3540-3549. Zbl 1216.47030 MR 2764897

[12] S. G. Krein, Yu. I. Petunin, and E. M. Semenov, Interpolation of linear operators. Transl. Math. Monographs. 54, Amer. Math. Soc., Providence, R.I., 1982. Zbl 0493.46058 MR 0649411

[13] S. Lord, A. Sedaev, and F. Sukochev, Dixmier traces as singular symmetric functionals and applications to measurable operators. J. Funct. Anal. 224 (2005), 72-106. Zbl 1081.46042 MR 2139105

[14] W. A. J. Luxemburg and B. de Pagter, Invariant means for positive operators and semigroups. In Circumspice. Various papers in and around mathematics in honour of Arnoud van Rooij. Nijmegen K. University Press, Nijmegen 2001, 31-55.

[15] J. R. Ringrose, Super-diagonal forms for compact linear operators. Proc. London Math. Soc. (3) 12 (1962), 367-384. Zbl 0102.10301 MR 0136998

[16] A. Sedaev, Geometrical and topological aspects of interpolation spaces of Petre's Kmethod (in Russian). Thesis, Voronezh 2010.

[17] A. Sedaev, F. Sukochev and D. Zanin, Lidskii-type formulae for Dixmier traces. Integral Equations Oper. Theory 68 (2010), 551-572. Zbl 1208.46062 MR 2745479

[18] F. Sukochev, A. Usachev and D. Zanin, Generalized limits with additional invariance properties and their applications to noncommutative geometry. Adv. Math. 239 (2013), 164-189. Zbl 06243625 MR 3045146

[19] F. Sukochev, A. Usachev and D. Zanin, On the distinction between the classes of Dixmier and Connes-Dixmier traces. Proc. Amer. Math. Soc. 141 (2013), 2169-2179. Zbl 1272.46057 MR 3034443 
[20] F. Sukochev and D. Zanin, $\zeta$-function and heat kernel formulae. J. Funct. Anal. 260 (2011), 2451-2482. Zbl 1235.46061 MR 2772378

Received December 15, 2011; revised April 30, 2012

F. Sukochev, School of Mathematics and Statistics, University of New South Wales, Sydney, 2052, Australia

E-mail: f.sukochev@unsw.edu.au

A. Usachev, School of Mathematics and Statistics, University of New South Wales, Sydney, 2052, Australia

E-mail: a.usachev@unsw.edu.au

D. Zanin, School of Mathematics and Statistics, University of New South Wales, Sydney, 2052, Australia

E-mail: d.zanin@unsw.edu.au 